\title{
A eficácia da terapia fotodinâmica no tratamento periodontal não cirúrgico
}

\author{
The effectiveness of photodynamic therapy \\ in non-surgical periodontal treatment \\ La eficacia de la terapia fotodinámica \\ en el tratamiento periodontal no quirúrgico \\ Camila Lima de OLIVEIRA ${ }^{1}$ \\ Kaiza de Sousa SANTOS ${ }^{2}$ \\ José de Alencar FERNANDES NETO \\ Ana Luzia Araújo BATISTA ${ }^{4}$ \\ Carmen Lúcia Soares Gomes de MEDEIROS ${ }^{5}$ \\ Maria Helena Chaves de Vasconcelos CATÃ ${ }^{6}$ \\ ${ }^{1}$ Graduanda em Odontologia, Departamento de Odontologia, Universidade Estadual da Paraíba, UEPB, \\ 58429-500, Campina Grande - PB, Brasil \\ ${ }^{2}$ Graduanda em Odontologia, Departamento de Odontologia, Universidade Estadual da Paraíba, UEPB, 58233-000, Araruna - PB, Brasil \\ Mestrando do Programa de Pós-Graduação em Odontologia, Departamento de Odontologia, \\ Universidade Estadual da Paraíba, UEPB, 58429-500, Campina Grande - PB, Brasil \\ ${ }^{4}$ Doutoranda do Programa de Pós-Graduação em Odontologia, Departamento de Odontologia, \\ Universidade Estadual da Paraíba, UEPB, 58429-500, Campina Grande - PB, Brasil \\ ${ }^{5}$ Professora Doutora em Odontologia, Departamento de Odontologia, Universidade Estadual da Paraíba \\ UEPB, 58429-500, Campina Grande - PB, Brasil \\ ${ }^{6}$ Professora Doutora do Programa de Pós-Graduação em Odontologia, Universidade Estadual da Paraíba, \\ UEPB, 58429-500, Campina Grande - PB, Brasil
}

\section{Resumo}

Introdução: A periodontia é uma especialidade odontológica que visa promover a saúde dos tecidos periodontais através do controle dos agentes etiológicos das periodontopatogenias, os microrganismos. Apesar da raspagem e alisamento coronoradicular consistir em um tratamento mecânico bastante utilizado, tal terapia não consegue proporcionar o controle dos patógenos nas áreas de difíceis instrumentação, motivo pelo qual torna-se necessário a utilização de terapias complementares que reduzam os redutos bacterianos em locais inacessíveis aos instrumentos periodontais. Objetivo: Relatar informações sobre o mecanismo de ação, aplicabilidade e eficácia da terapia fotodinâmica no tratamento periodontal não cirúrgico. Material e método: Foi realizado uma revisão de literatura conduzida através do acesso às bases de dados eletrônicas Pubmed, Scielo, Lilacs e Periódicos Capes. Como estratégica de busca utilizou-se as palavras chaves "Laser", "Photodynamic Therapy" e "Periodontal Disease". Resultados: Redução bacteriana e efeitos colaterais mínimos são vantagens atribuídas à terapia fotodinâmica, no entanto, a literatura evidencia estudos que apresentam resultados controversos quanto ao efeito adicional da terapia fotodinâmica no tratamento periodontal. Conclusão: É necessária a padronização dos protocolos empreendidos para que se obtenha dados mais esclarecedores sobre a eficácia da terapia fotodinâmica no tratamento periodontal não cirúrgico.

Descritores: Fotoquimioterapia; Lasers; Terapia com Luz de Baixa Intensidade; Doenças Periodontais; Periodontia.

\begin{abstract}
Introduction: Periodontics is a dental specialty that aims to promote the health of periodontal tissues by controlling the etiological agents of periodontopathogens, microorganisms. Although the scraping and straightening of the coronary artery consists of a well-used mechanical treatment, such therapy cannot provide the control of the pathogens in areas of difficult instrumentation, which is why it is necessary to use complementary therapies that reduce bacterial retrusions in places inaccessible to Periodontal instruments. Objective: To report information on the mechanism of action, applicability and efficacy of photodynamic therapy in non-surgical periodontal treatment. Material and method: A literature review was performed using a set of articles in Pubmed, Scielo, Lilacs and Capes Periodicals databases. As a search strategy, the key words Laser, Photodynamic Therapy and Periodontal Disease were used. Results: Bacterial reduction and minimal side effects are advantages attributed to photodynamic therapy, however, the literature shows studies that present controversial results regarding the additional effect of photodynamic therapy in the periodontal treatment. Conclusion: It is necessary to standardize the protocols undertaken to obtain more enlightening data on the efficacy of photodynamic therapy in non-surgical periodontal treatment. Descriptors: Photochemotherapy; Lasers; Low-Level Light Therapy; Periodontal Diseases; Periodontics.
\end{abstract}

\section{Resumen}

Introducción: Periodoncia es una especialidad de la odontología que tiene como objetivo promover la salud de los tejidos periodontales mediante el control de los agentes etiológicos de periodontopatogenias, microorganismos. Aunque el raspado y alisado coronoradicular consistir en un tratamiento mecánico ampliamente utilizado, este tipo de tratamiento no puede proporcionar control de microorganismos en las zonas de difícil instrumentación, por lo que se hace necesario el uso de terapias adicionales que reducen reductos bacterianas en lugares inaccesibles a instrumentos periodontales. Para informar de información sobre el mecanismo de acción, la aplicabilidad y eficacia de la terapia fotodinámica en el tratamiento periodontal no quirúrgico. Métodos: Una revisión de la literatura se realizó usando un conjunto de artículos en Pubmed, Scielo, Lilacs y Portal Periodicos Capes. Como se utilizó la estrategia de búsqueda de palabras clave con láser, la terapia fotodinámica y la enfermedad periodontal. Resultados: La reducción bacteriana y efectos secundarios mínimos son ventajas conferidas terapia fotodinámica, sin embargo, la literatura muestra que los estudios tienen resultados contradictorios sobre el efecto adicional de la terapia fotodinámica en el tratamiento periodontal. Conclusión: Es necesaria la estandarización de los protocolos emprendidos para que se obtengan datos más claros sobre la eficacia de la terapia fotodinámica en el tratamiento periodontal no quirúrgico.

Descriptores: Fotoquimioterapia; Rayos Láser; Terapia por Luz de Baja Intensidad; Enfermedades Periodontales; Periodoncia.

\section{INTRODUÇÃO}

A doença periodontal é uma infecção local que afeta gengiva, cemento, ligamento periodontal e osso alveolar, ocasionada por bactérias patogênicas do biofilme oral, que se adere ao sulco gengival de hospedeiros susceptíveis.
A liberação de enzimas, endotoxinas e fatores citotóxicos pelos patógenos desencadeiam mecanismos de inflamação no periodonto, capazes de ocasionar diversas alterações nos tecidos de proteção e sustentação do elemento dentário ${ }^{1}$. 
A raspagem e alisamento coronoradicular (RAR) é considerada o método mais eficaz no tratamento das doenças periodontais, atuando na eliminação de microrganismos localizados sub e supragengival, cálculo, cemento e dentina contaminada. Aparelhos sônicos e ultrassônicos foram desenvolvidos com o intuito de potencializar as melhorias proporcionadas pelos instrumentos periodontais manuais, no entanto, ambas as técnicas não demonstraram total efetividade na eliminação dos agentes etiológicos na região subgenvival ${ }^{2}$.

Quando o processo inflamatório não é solucionado através da raspagem e alisamento coronoradicular, a antibioticoterapia pode ser utilizada como coadjuvante ao tratamento periodontal mecânico. Apesar de proporcionarem redução de microrganismos presentes na bolsa periodontal, fatores limitantes bem como efeitos adversos são características atribuídas aos antibióticos, motivo pelo qual estudos tem analisado de forma criteriosa a prescrição de tais substâncias no controle das doenças periodontais ${ }^{3}$.

A terapia fotodinâmica (PDT) consiste em uma abordagem terapêutica bastante utilizada na área da saúde. $\mathrm{Na}$ Odontologia, os lasers de baixa potência são testados em virtude do seu efeito bactericida, analgésico e hemostático. $\mathrm{Na}$ tentativa de proporcionar melhorias clínicas aos pacientes, a periodontia tem demonstrado interesse por terapias complementares que correlacionem redução bacteriana e efeitos colaterais mínimos, motivo pelo qual a terapia fotodinâmica vem sendo estudada como alternativa ao tratamento periodontal não cirúrgico ${ }^{4}$.

Considerando a relevância da terapia fotodinâmica no contexto do tratamento periodontal, o objetivo desse trabalho é explorar a literatura dando enfoque ao mecanismo de ação e aplicabilidade da PDT no tratamento periodontal não cirúrgico, estabelecendo através de resultados obtidos em ensaios clínicos, microbiológicos e em animais, uma discussão sobre a eficácia desta terapia no tratamento periodontal não cirúrgico.

\section{MATERIAL E MÉTODO}

Para alcançar o objetivo proposto, realizou-se uma revisão de literatura conduzida através do acesso às bases de dados eletrônicas Pubmed, Scielo, Lilacs e Periódicos Capes, utilizando isoladamente ou em associação as palavras-chaves "Laser", "Photodynamic Therapy" e "Periodontal Disease". A coleta de dados ocorreu em novembro de 2016.

Os trabalhos científicos encontrados de acordo com a estratégica de busca estabelecida foram posteriormente submetidos à triagem inicial. Inicialmente realizou-se a filtragem dos artigos que foram publicados em revistas científicas no período de 2000 a 2016, seguido pela exclusão (por meio da leitura dos títulos e resumos) dos estudos que não apresentassem relação com o tema proposto. Na triagem secundária os artigos pré-selecionados foram lidos na íntegra e submetidos a uma criteriosa avaliação, que levou em consideração os seguintes aspectos: procedência das revistas, indexações, detalhamento metodológico e consistência dos resultados apresentados pelos autores. Posteriormente a análise, os artigos científicos foram classificados como elegíveis (estudos relevantes para o tema, com possibilidade de serem inclusos na revisão) e não elegíveis (estudos irrelevantes para o tema, sem possibilidade de serem inclusos na revisão).

Como critérios de inclusão do levantamento utilizaram-se: período de publicação, relação com o tema proposto, significância estatística e confiabilidade dos resultados dos estudos. Foram excluídos todos os artigos científicos que não se enquadraram nos critérios de inclusão. Dos artigos encontrados, 49 foram pré-selecionados e 29 foram incluídos na revisão.

\section{REVISÃO DA LITERATURA}

A inflamação gengival, migração do epitélio juncional e exposição radicular caracterizam as lesões periodontais iniciais. Destruição do ligamento periodontal, reabsorção óssea e mobilidade dental são consequências da periodontite, uma das grandes causas de perda dentária nos adultos. Os benefícios proporcionados pelo tratamento periodontal mecânico convencional podem ser verificados clinicamente, onde redução do sangramento à sondagem, estabilização dos níveis de inserção clínica e diminuição da profundidade clínica sondagem foram melhorias frequentemente relatadas nos estudos que propuseram verificar os parâmetros clínicos de pacientes submetidos à terapia periodontal não-cirúrgica ${ }^{5}$.

Aspectos relacionados ao hospedeiro bem como características dos patógenos periodontais são evidenciados como fatores que limitam a eficácia do tratamento promovido pela RAR. Anatomia dentária complexa (região de furca, concavidades, sulcos, sítios distais de molares), bolsas de trajetos sinuosos, bem como a capacidade de alguns microrganismos (Aggregatibacter actynomycetencomitans, Porphyromonas gingivalis) invadirem tecidos moles, são aspectos que contribuem para a recolonização de sítios anteriormente tratados, em consequência da permanência de redutos bacterianos em locais inacessíveis aos instrumentos periodontais ${ }^{6}$.

A PDT envolve o uso de um laser de baixa potência com comprimento de onda adequado para eliminar microrganismos tratados com drogas fotosensibilizadoras. $\mathrm{O}$ processo se baseia em um conjunto de reações fotooxidativas, que ao desencadear alterações morfobiológicas, promovem necrose celular. $\mathrm{Na}$ periodontia, o agente fotossensibilizador é impregnado no biofilme subgengival, penetrando nas células bacterianas. A emissão da fonte de luz dos lasers ocasiona excitação do corante, desencadeando reações químicas que promovem geração de energia. O oxigênio das células bacterianas ao absorver a energia liberada transforma-se em oxigênio singleto, que juntamente com radicais livres altamente reativos, degradam polissacarídeos e destroem os sistemas biológicos bacterianos $^{7}$.

Os lasers são classificados em dois grupos: baixa potência e alta potência. $\mathrm{Na}$ odontologia, os de baixa potência são utilizados em maior frequência (tanto na clínica como em pesquisas científicas), em virtude de apresentarem melhor custo-benefício. Dentre esses, os mais conhecidos e estudados são os de diodo Gálio-Alumínio-Arsênio (GaAlAs ou GaAs), com comprimento de onda variando de 660 a 909nm e os de Hélio-Neônio (HeNe), com comprimento de onda de $632 \mathrm{~nm}^{8}$.

Os corantes fenotiazínicos são os mais comumente utilizados em odontologia. Os agentes fotossensibilizadores dessa classe apresentam fototoxicidade tanto ao núcleo quando às membranas celulares. O mais conhecido deles é o azul de metileno (AM), cuja máxima absorção ocorre em $664 \mathrm{~nm}$, ou seja, a PDT com o AM deve utilizar fontes de luz emitindo fótons na faixa do vermelho visível, como os lasers vermelhos de baixa potência e os LEDs vermelhos ${ }^{9}$.

Outro fator importante com relação ao agente 
fotossensibilizador é a sua concentração. $\mathrm{Na}$ periodontia deve-se optar pelo azul de metileno a $0,01 \%$ porque a concentração de $0,005 \%$ é indicada em casos onde não haja exsudato, sangue, fluido gengival, saliva ou qualquer outro tipo de diluente ou conteúdo proteico, como canais radiculares e superfície dental (após o preparo protético ou cavitário) ${ }^{9}$.

Efeito analgésico, anti-inflamatório e biomodulador são alguns dos benefícios atribuídos pela literatura à PDT. Além de não necessitar da realização de anestesia, a terapia despertou o interesse da periodontia em virtude das seguintes características: efeito bactericida em curto período de tempo, aceleração da reparação tecidual, redução da necessidade de realizar retalhos, diminuição do tempo de tratamento, redução de inflamações crônicas, aceleração de cicatrização e mínimos efeitos colaterais e sistêmicos ${ }^{4}$.

Para promover redução bacteriana sem causar efeitos tóxicos graves aos pacientes, os lasers de baixa potência precisam ser utilizados com cautela, devendo os cirurgiõesdentistas ficarem atentos quanto os seguintes critérios: tempo de aplicação, níveis de energia e local de emissão. O uso inadequado pode ocasionar crateras radiculares e destruição do aparato de inserção sadio do fundo das bolsas periodontais ${ }^{10}$. A aplicação do agente fotossensibilizador sem a emissão da fonte luz não promove danos aos tecidos sadios, no entanto, o processo de destruição bacteriana só acontecerá quanto o corante fotossensível for utilizado em associação a fonte de luz ${ }^{11}$.

\section{DISCUSSÃO}

Verificar a possibilidade de substituição dos antibióticos por PDT, consistiu em objetivos da pesquisa de Arweiler et al. ${ }^{12}$, no entanto, a antibioticoterapia apresentou melhores resultados.

A azitromicina, metronidazol e doxiciclina foram antibióticos testados na pesquisa de Haffajee et al. ${ }^{13}$, na qual resistência bacteriana e incapacidade de penetração na matriz extracelular do biofilme dental foram evidenciados como características dos antibióticos. Distúrbios gastrointestinais, alergias, absorção de tetraciclinas em ossos e dentes, artropatias, dores de cabeça, tonturas, gosto metálico ou intolerância ao álcool, foram efeitos adversos verificados em pacientes que fizeram uso da antibioticoterapia sistêmica como forma de tratamento. Tal aspecto revela a necessidade da inserção de terapias complementares mais eficazes, que reduzam os patógenos sem causar efeitos colaterais graves ${ }^{14}$.

$\mathrm{O}$ interesse de pesquisadores em testar a eficácia de antibióticos locais como coadjuvantes ao tratamento periodontal não cirúrgico, ocorreu em virtude dos resultados limitantes obtidos pela antibioticoterapia sistêmica. Apesar de ocasionarem menores efeitos adversos, um conjunto de desvantagens foi atribuído aos antimicrobianos locais (plena adesão do paciente ao tratamento, custo e aplicabilidade em pequenas áreas da boca), que somada a inconsistência de resultados clínicos, não fornecem total credibilidade a utilização de tais medicamentos ${ }^{15}$.

No que se refere ao tratamento periodontal não cirúrgico, estudos científicos buscaram avaliar a eficácia da terapia fotodinâmica como coadjuvante ao tratamento periodontal mecânico convencional. Estudos in vitro, in vivo e em animais caracterizam os tipos de metodologias utilizadas para alcance do objetivo proposto, onde variância de protocolos metodológicos (tipo de laser, concentração do fotossensibilizador, densidade de energia, potência, comprimento de onda e tempo de aplicação) são evidenciadas como limitações dos estudos presentes até o momento ${ }^{16}$

Diversos estudos in vitro tem comprovado alta taxa de destruição de microrganismos submetidos à terapia fotodinâmica ${ }^{17}$. A PDT usando visible light plus waterfiltered infrared-A wavelengths (VIS e wIRA) em combinação com o fotossensibilizador cloro e6 (Ce6), exibiram alta atividade antimicrobiana contra Streptococcus mutans, Enterococcus faecalis e o biofilme supragengival formado $^{18}$.

Além de comprovar que a ação bactericida foi maior quando laser $\mathrm{e}$ fotossensiblizador foram aplicados conjuntamente $^{19}$, estudos evidenciaram a interferência do comprimento de onda dos lasers nos resultados obtidos. Na pesquisa de Chan et al. ${ }^{20}$, a maior eliminação de bactérias ocorreu com o laser diodo GaAlAs $(665 \mathrm{~nm} ; 100 \mathrm{~mW} ; 21,2$ $\mathrm{J} / \mathrm{cm}^{2} ; 60$ segundos), quando comparado ao GaAlAs (830nm;100mW; 21,2 J/cm²; 60 segundos)

Apesar da PDT exercer efeito antimicrobiano tanto em bactérias gram-positivas quanto em gram-negativas, a literatura evidencia que quanto menor a complexidade da membrana externa, maior será a facilidade de destruição celular. Em virtude de apresentarem lipoproteínas e lipopolissacarídeos na constituição celular, bactérias periodontopatogênicas gram-negativas dificultam a inativação fotoquímica ${ }^{21}$. Na pesquisa de Pfitzner et al. ${ }^{22}$ a bactéria Aggregatibacter actynomycetencomitans demonstrou maior resistência ao efeito da terapia.

Redução bacteriana e menor perda óssea histológica foram resultados verificados em estudos científicos, nos quais ratos foram submetidos à PDT em associação com a raspagem. Os tipos de fotossensibilizadores bem como os lasers testados foram respectivamente: toluidina com laser $630 \mathrm{~nm}$ e azul de com laser $660 \mathrm{~nm}^{19}$. A terapia também foi satisfatória em pesquisas realizadas com ratos diabéticos ${ }^{23} \mathrm{e}$ ratas ovariectomizadas ${ }^{24}$. Nos estudos mencionados a raspagem promoveu redução da perda óssea histológica, no entanto, os resultados foram menos significativos quanto comparado a eficácia promovida pela terapia fotodinâmica. $\mathrm{Na}$ pesquisa de Sorkhdini el al. ${ }^{25}$ cães com doença periodontal induzida foram tratados com raspagem e terapia fotodinâmica (clorina e6 associado ao laser de 662nm). No estudo em questão a PDT não proporcionou resultados significativos e não houve diferenças entre os grupos de tratamento.

A eficácia da terapia no tratamento periodontal é analisada através dos seguintes parâmetros clínicos: sangramento a sondagem, níveis de inserção clínica, profundidade de sondagem e recessão gengival. Em alguns estudos verificou-se que a PDT proporcionou resultados clínicos significativos, reduzindo tanto o sangramento à sondagem $^{26}$, como a profundidade de sondagem ${ }^{27}$. Na pesquisa de Moreira et al. ${ }^{28}$, além dos benefícios obtidos em relação aos aspectos mencionados anteriormente, verificouse também ganho de inserção clínica nos pacientes analisados. Fato contrário foi observado em outros trabalhos científicos, onde não foi mencionado ganhos significativos em relação aos parâmetros clínicos avaliados ${ }^{29}$.

\section{CONCLUSÃO}

Apesar das doenças periodontais não serem solucionadas unicamente pelo uso da PDT, diversos estudos a evidenciaram como coadjuvante eficaz no tratamento periodontal não cirúrgico, podendo ser utilizada também como alternativa à antibioticoterapia. A ausência de padronização metodológica bem como o fato de ser aplicada 
adjuvante ou não a RAR contribui para a existência de conclusões controversas para o tema em questão.

É necessária a padronização nos parâmetros e protocolos empreendidos, para que ao reduzir as carências metodológicas, aumente-se a confiabilidade dos estudos que se propõem a avaliar a eficácia da PDT no tratamento das doenças periodontais.

\section{REFERÊNCIAS}

1. Malik R, Manocha A, Suresh, DK. Photodynamic therapy-A strategic review. Indian J Dent Res. 2010; 21(2):285-91.

2. Loos BG, John RP, Laine ML. Identification of genetic risk factors for periodontitis and possible mechanisms of action. J Clin Periodonto. 2005; 32(6):159-79.

3. Herrera D, Alonso B, León R, Roldán S, Sanz M. Antimicrobial therapy in periodontitis: the use of systemic antimicrobials against the subgingival biofilm. J Clin Periodonto. 2008; 35(8 Suppl):45-66.

4. Ishikawa I, Aoki A, Takasaki AA, Mizutani K, Sasaki KM, Izumi,Y. Application of lasers in periodontics: true innovation or myth? Periodontology. 2009;50(1):90-126.

5. Tsai CY, Tang CY, Tan TS, Chen KH, Liao KH, Liou ML. Subgingival microbiota in individuals with severe chronic periodontitis. Braz. J Microbiol Immunol Infect. 2016; 4(3):1-9.

6. Rajesh S, Koshi E, Philip K, Mohan A. Antimicrobial photodynamic therapy: An overview. J Indian Soc Periodontol. 2011; 15(4): 323-7.

7. Fernandes LA, De Almeida JM, Theodoro LH, Bosco AF, Nagata MJH, Martins TM, et al. Treatment of experimental periodontal disease by photodynamic therapy in immunosuppressed rats. J Clin Periodonto. 2009; 36(3):219-28.

8. Luchesi VH, Pimentel S, Kolbe MF, Ribeiro F, Casarin $\mathrm{RC}$, Nociti FH, et al. Photodynamic therapy in the treatment of class II furcation: a randomized controlled clinical trial. J Clin Periodonto. 2013;40(8):781-88.

9. Harris F, Chatfield LK, Phoenix, DA. Phenothiazinium based photosensitisers-photodynamic agents with a multiplicity of cellular targets and clinical applications. Curr Drug Targets. 2005; 6(5):615-27.

10. Schwarz F, Aoki A, Becker J, Sculean A. Laser application in non-surgical periodontal therapy: a systematic review. J Clin Periodonto. 2008; $35(8$ Suppl): 29-44.

11. Al Habashneh R, Asa'ad FA, Khader Y. Photodynamic therapy in periodontal and peri-implant diseases. Quintessence Int. 2015; 46(8):670-7.

12. Arweiler NB, Pietruska M, Pietruski J, Skurska A, Dolińska E, Heumann $\mathrm{C}$, et al. Six-month results following treatment of aggressive periodontitis with antimicrobial photodynamic therapy or amoxicillin and metronidazole. Clin Oral Investig. 2014; 18(9):2129-35.

13. Haffajee AD, Torresyap G, Socransky, SS. Clinical changes following four different periodontal therapies for the treatment of chronic periodontitis: 1-year results. J Clin Periodonto. 2007; 34(3):243-53.

14. Ryder MI. An update on HIV and periodontal disease. J. Periodontol. 2002; 73(9):1071-8.

15. Azarpazhooh A, Shah PS, Tenenbaum HC, Goldberg MB. The effect of photodynamic therapy for periodontitis: a systematic review and meta-analysis. J. Periodontol. 2010; 81(1):4-14.

16. Balata ML, Ribeiro ÉDP, Bittencourt S, Tunes UDR. Terapia fotodinâmica como adjuvante ao tratamento periodontal não cirúrgico. Periodontia. 2010; 20(2):22-32.

17. Sigusch BW, Pfitzner A, Albrecht V, Glockmann E. Efficacy of photodynamic therapy on inflammatory signs and two selected periodontopathogenic species in a beagle dog model. J. Periodontol. 2005; 76(7):1100-5.

18. Karygianni L, Ruf S, Follo M, Hellwig E, Bucher M, Anderson AC, et al. Novel broad-spectrum antimicrobial photoinactivation of in situ oral biofilms by visible light plus water-filtered infrared A. Applied and environmental microbiology. 2014; 80(23):7324-36.

19. Prates RA, Yamada AM, Suzuki LC, Hashimoto MCE, Cai S, Gouw-Soares S, et al. Bactericidal effect of malachite green and red laser on Actinobacillus actinomycetemcomitans. J Photochem Photobiol B. 2007; 86(1):70-6.

20. Chan Y, Lai CH. Bactericidal effects of different laser wavelengths on periodontopathic germs in photodynamic therapy. Lasers Med Sci. 2003; 18(1):51-5.

21. Usacheva MN, Teichert MC, Biel MA. Comparison of the methylene blue and toluidine blue photobactericidal efficacy against gram-positive and gram-negative microorganisms. Lasers Surg Med. 2001; 29(2):165-73.

22. Pfitzner A, Sigusch BW, Albrecht V, Glockmann E. Killing of periodontopathogenic bacteria by photodynamic therapy. J. Periodontol. 2004; 75(10): 1343-9.

23. de Almeida, JM, Theodoro LH, Bosco AF, Nagata MJH, Oshiiwa M, Garcia, VG. In vivo effect of photodynamic therapy on periodontal bone loss in dental furcations.J. Periodontol. 2008; 79(6): 1081-88.

24. Garcia VG, Gualberto Júnior EC, Fernandes LA, Bosco AF, Hitomi Nagata MJ, Casatti CA, et al. Adjunctive antimicrobial photodynamic treatment of experimentally induced periodontitis in rats with ovariectomy. J Periodontol. 2013; 84(4):556-65.

25. Sorkhdini P, Moslemi N, Jamshidi S, Jamali R, Amirzargar AA, Fekrazad R. Effect of Hydrosoluble Chlorine-Mediated Antimicrobial Photodynamic Therapy on Clinical Parameters and Cytokine Profile in Ligature-Induced Periodontitis in Dogs. J Periodontol. 2013; 84(6):793-800.

26. Theodoro LH, Silva SP, Pires JR, Soares GHG, Pontes AEF, Zuza EP, et al. Clinical and microbiological effects of photodynamic therapy associated with nonsurgical periodontal treatment. A 6-month follow-up. Lasers Med Sci. 2012; 27(4):687-93.

27. Bassir SH, Moslemi N, Jamali R, Mashmouly S, Fekrazad R, Chiniforush N, et al. Photoactivated disinfection using light-emitting diode as an adjunct in the management of chronic periodontitis: a pilot double-blind split-mouth randomized clinical trial. J Clin Periodonto. 2013; 40(1):65-72.

28. Moreira AL, Novaes Jr AB, Grisi MF, Taba Jr M, Souza SL, Palioto DB, et al. Antimicrobial photodynamic therapy as an adjunct to non-surgical treatment of aggressive periodontitis: a split-mouth randomized controlled trial. J Periodontol. 2015; 86(3):376-86.

29. Pourabbas R, Kashefimehr A, Rahmanpour N, Babaloo Z, Kishen A, Tenenbaum HC, et al. Effects of photodynamic therapy on clinical and gingival crevicular fluid inflammatory biomarkers in chronic periodontitis: a split-mouth randomized clinical trial. J Periodontol. 2014; 85(9):1222-9. 


\section{CONFLITO DE INTERESSES}

Os autores declaram não haver conflitos de interesse.

\section{AUTOR PARA CORRESPONDÊNCIA}

Maria Helena Chaves de Vasconcelos Catão mhelenact@zipmail.com.br

Submetido em 24/03/2017 Aceito em 02/05/2017 